\title{
Instantaneous Deformation Monitoring Research on the Racks in the Dense Channels Accessing-Sorting System
}

\author{
Yongqian $\mathrm{Zhao}^{1}$, Yaohua $\mathrm{Wu}^{2}$ and Chengxin $\mathrm{Yu}^{3}$ \\ ${ }^{1,2}$ School of Control Science and Engineering, Shandong University, \\ Jinan 250061, China \\ ${ }^{1}$ School of Natural Science, Shandong Jianzhu University, Jinan 250101, China \\ ${ }^{3}$ Assets Department, Shandong Jianzhu University, Jinan 250101, China \\ 13455111419@163.com, ${ }^{2}$ Yaohua.Wu@sdu.edu.cn, ${ }^{3}$ ycx1108@126.com
}

\begin{abstract}
With the prosperity of e-commerce and the sharper increasing orders, the requirements of the racks' height in automatic warehouse and the weight that it can bear is higher and higher. The Dense Channels Accessing-Sorting System (Shorted as: DCASS) is a new kind of the logistic system that can be used well in the e-commerce logistic centers. For the potential hazard of racks in DCASS will be very fatal to the logistics enterprises, it is an urgent task to monitor the instantaneous deformation of racks. This essay gives an empirical research with Digital Close-Range Photogrammetric (Shorted as:DCRP) method to this problem. In the experiment, we take photos with digital cameras, and use digital analytical method to process the data that collected with computers. For the processed data has reduced the influence of outside condition and the instability of internal-external azimuth from digital cameras effectively, we get the result with precision up to $2.3 \%$.
\end{abstract}

Keywords: DCRP method, GM $(1,1)$, racks, DCASS, monitoring

\section{Introduction}

Due to the increasing scarcity of human operators and the fast development of ecommerce, there is a trend towards higher levels of automation in logistic centers. As the very import part in the DCASS, racks are used for storage and load products [1]. It will be huge invested capital in warehouse development project. High-level racks play an important role on saving land, improving storage density, and reducing labor intensity [23]. To avoid the loss and safety hazard caused by deformation of racks, we make a feasible solution to monitor the racks instantaneously, dynamically and continuously. In this essay, we take DCRP method to monitor the deformation of racks in the DCASS, which has been used in a vast field successfully.

Considerable developments in design and operations of different types of storage systems have taken place in the last two decades, and many important models and review papers have appeared in the literature. Hassini [4-5], Litvak and Vlasiou [6] and Hassini and Vickson [7] extensively reviewed the literature and presented a general model that includes one- and two-dimensional carousel storage location problems, as well as other related problems. A carousel can be classified as an automated storage and retrieval system, which is widely used in warehousing or manufacturing. It consists of a number of racks or drawers rotating in a closed loop, operated by an on-board computer that automatically registers stock locations and items stocked, with automatic retrieval, and a picker in front of the carousel. Being made of a huge variety of configurations, sizes, and types, the carousel systems can be set horizontal or vertical, and rotate in either one or both directions. While carousel systems show space saving as a major benefit, they suffer 
from complexity in replenishments. Different from above, we will develop a series of experiment to explore the security and stability of the racks in the automated storage and retrieval systems, especially the DCASS.

As we systematically integrate digital cameras and computer to obtain, store, process, manage data and execute other duties, the speed of processing data is doubled. The images are stored stably and fixed color, which have the advantages of non-treatment, automation, real time and so on [8-9]. In the experiment, we take photos for racks monitored continuously by four digital cameras, the images are transported into computer and processed automatically, and the results will be displayed on screen, then we can get the deformation details of racks timely. It will benefit for the DCASS greatly by using our method especially when it is running. For the DCRP method can be used to monitor deformation of racks successfully in the normal weather and working conditions, and its simple and cheap devices will be helpful to improve the utilization and safety of highlevel automatic warehouse, so it will be widely accepted by logistics enterprises.

In this paper, we will discuss following problems mainly:

1. The Grey Models and DCRP methods are put forward and are used in the experiments.

2. Combine with the deformation process of racks in the DCASS, the experiment data is collected and dealt with.

3. To explore a satisfying conclusion and get a result with precision to $2.3 \%$.

\section{Research Models}

\subsection{Grey Model}

The grey theory model is a convenient method for deformation analysis; we get some satisfactory results with a little data information by using $\mathrm{GM}(1,1)$ and $\mathrm{GM}(2,1)$ model. The differential equations established according to the grey system theory are named GM model $[10,11]$. The modeling process and principle of GM $(1,1)$ is as follows.

Let non-negative sequence $X_{0}=\left\{x_{0}(1), x_{0}(2), \cdots, x_{0}(n)\right\}, x_{0}(k) \geq 0, k=1,2, \cdots, n$, its generating data sequence $X_{1}=\left\{x_{1}(1), x_{1}(2), \cdots, x_{1}(n)\right\}$, in which,

$$
x_{1}(k)=\sum_{l=1}^{k} x_{0}(l), k=1,2, \cdots, n .
$$

Then the sequence $x_{1}$ was first-order accumulated generating sequence of $x_{0}$, named Accumulated Generating Operator denoted as 1-AGO.

Let $Z_{1}=\left\{z_{1}(1), z_{1}(2), \cdots, z_{1}(n)\right\}$ be the tight-neighbor mean value generation sequence of $X_{1}$, in which,

$$
z_{1}(k)=\frac{x_{1}(k)+x_{1}(k-1)}{2}, k=2,3, \cdots, n .
$$

then we get:

GM $(\mathbf{1}, \mathbf{1}): x_{0}(k)+a z_{1}(k)=b$

In which, $a, b$ can be computed from $(a, b)^{T}=\left(B^{T} B\right)^{-1} B^{T} Y$, and we have: 


$$
Y=\left\{\begin{array}{c}
x_{0}(2) \\
x_{0}(3) \\
\vdots \\
x_{0}(n)
\end{array} \mid, B=\left\{\begin{array}{cc}
-z_{1}(2) & 1 \\
-z_{1}(3) & 1 \\
\vdots & \vdots \\
-z_{1}(n) & 1
\end{array}\right]\right.
$$

Then we call $\frac{d x_{1}}{d t}+a x_{1}=b$ as grey differential equation, and the albino equation of the equation $x_{0}(k)+a z_{1}(k)=b$ also named as shadow equation. The solution is:

$$
\hat{x}_{1}(k+1)=\left(x_{1}(0)-\frac{b}{a}\right) e^{-a k}+\frac{b}{a}, \quad k=1,2, \cdots, n
$$

We also have:

$$
\hat{x}_{0}(k+1)=\hat{x}_{1}(k+1)-\hat{x}_{1}(k), \quad k=1,2, \cdots, n
$$

\subsection{DCRP Method}

Digital Close-Range Photogrammetric (DCRP) method for adjusting digital camera is a method to establish the direct linear relation between coordinate and space position of observation points, which deal with the difference between internal-external azimuth of photos and map scale, and adjust its distortion errors. In the experiment, we usually set 16 monitoring points indoors [12]. Make the direct linear transformation as basic formulation; and the monitor points coordinate as the observation value with weight, correlative formulas of $\mathrm{L}$ coefficients as restrictive conditions, and indirect adjustment method with restricted conditions as adjust method of solution.

In case of given conditions, we have following formula:

$$
\left\{\begin{array}{c}
x-\frac{L_{1} X+L_{2} Y+L_{3} Z+L_{4}}{L_{9} X+L_{10} Y+L_{11} Z+1}=0 \\
z-\frac{L_{5} X+L_{6} Y+L_{7} Z+L_{8}}{L_{9} X+L_{10} Y+L_{11} Z+1}=0
\end{array}\right.
$$

In the upper formula, $x$ and $z$ are coordinates without errors, which is a sufficient and necessary condition to create the relation between $L$ coefficients and internal-external azimuth. After adjust the deformation of photograph negative, we can obtain the $L$ coefficients and the approximate values of the main photograph coordinate. By monitoring points with direct linear transformation formula we can correct object distortion.

After we eliminate the system errors like the negative deformation and the object distortion, we can establish linearization of linear transformation error equation for the observation of image points coordinate. Then we have the formula:

$$
\left(\begin{array}{c}
P_{1} \\
P_{2}
\end{array}\right) V=\left(\begin{array}{l}
V_{1} \\
V_{2}
\end{array}\right)=\left(\begin{array}{ll}
M & N \\
0 & I
\end{array}\right)\left(\begin{array}{l}
\Delta L \\
\Delta X
\end{array}\right)-\left(\begin{array}{l}
W_{1} \\
0
\end{array}\right)
$$

In which, $\Delta L$ is the correction value of monitor point coordinate; ${ }_{N}$ is the coefficients of $\Delta L ; W_{1}$ is constant of error equation for observation value of image points; $P_{1}$ is the observation weight value of image points; and $P_{2}$ is the observation coordinate weight value of monitor points. 
In the direct linear transformation formula, there are 2 correlative formulas of $11 \mathrm{~L}$ coefficients. Take the two formulas as restrictive conditions when solving, then we can get the equation below:

$$
A \Delta L+W_{2}=0
$$

In which, $\mathbf{A}$ is the restrictive condition coefficient; $w_{2}$ is the restrictive condition discrepancy. From equation (8) and (9), we can get:

$$
\left.\left\{\begin{array}{lll}
M^{T} N & M^{T} N & A^{T} \\
N^{T} M & N^{T} N+P_{2} & 0 \\
A & 0 & 0
\end{array}\right)\left(\begin{array}{l}
\Delta L \\
K
\end{array}\right) \begin{array}{l}
M^{T} W_{1} \\
K X
\end{array} \mid \begin{array}{l}
N^{T} W_{1} \\
-W_{2}
\end{array}\right)=0
$$

We take weight coefficient method to accesses the precision of any monitor elements.

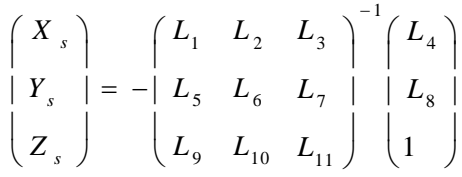

$$
\begin{aligned}
& m_{i}=M_{0} \sqrt{Q_{i j}}
\end{aligned}
$$

In which, $M_{0}$ is the unit weights error; $Q_{i j}$ is the weight coefficient of monitor elements.

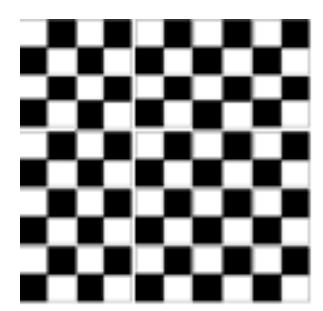

Figure 1. A kind of Cross Grid Glasses
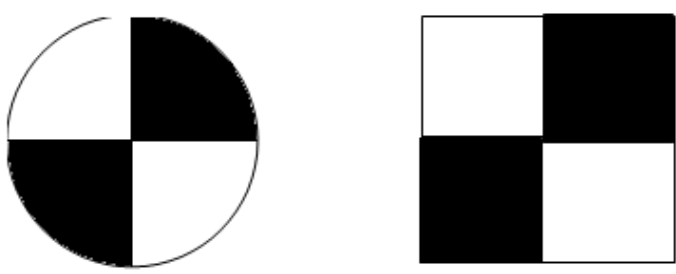

Figure 2. Images of Deformation Mark (left) and ReferenceMark (Might)

In order to improve the monitor precision of digital cameras, we took cross grid glasses which was made by ourselves, such as Figure 1, then we allocated the image points at the image coordinate system which have used the centre point of the cross grid as the origin, so as to calculate film deformation and objective distortion correctly.

This experiment shows that it is more universal than other methods to take direct linear transformation formula.

\section{Two Experiments' Processes}

In the twice experiments we make, the first one is under static pressure, and the second one is under dynamic condition. The first one is easy to operate, and there is fewer data to deal with; the second one is difficult to do relatively, also there is larger and complex data to process.

\subsection{Static Pressure Experiment}

The experiment devices are took two digital cameras which have been adjusted and one computer, and two computers installed software system which have developed by us and a printer to get the experiment results. The racks are made up of angle steel in the 
DCASS as model, such as Figure 3 and Figure 4. The designed yield pressure of the rack is $140 \mathrm{KN}$.

Put pressure of $50 \mathrm{KN}, 60 \mathrm{KN}, 70 \mathrm{KN}, 80 \mathrm{KN}, 100 \mathrm{KN}, 120 \mathrm{KN}$ on the racks step by step at first, and then reduce the pressure to $90 \mathrm{KN}, 80 \mathrm{KN}, 60 \mathrm{KN}$, and give records on a board set near the rack.

In the experiment, the two digital cameras are $8 \mathrm{~m}$ and $9 \mathrm{~m}$ far from the rack model, and take photos when the pressure is up to the designed value, and then input the digital image into computer in time. And the software in the computer will process it quickly.

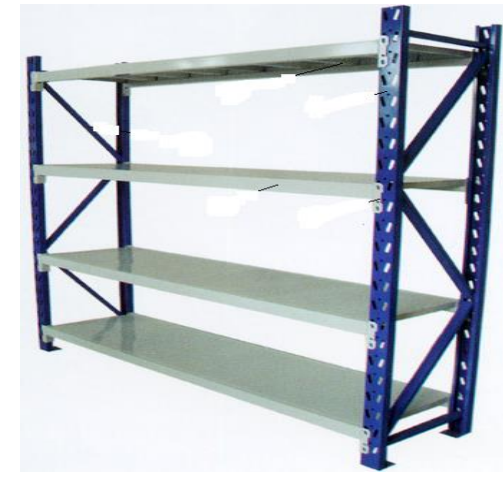

Figure 3. The Rack for the First Experiment

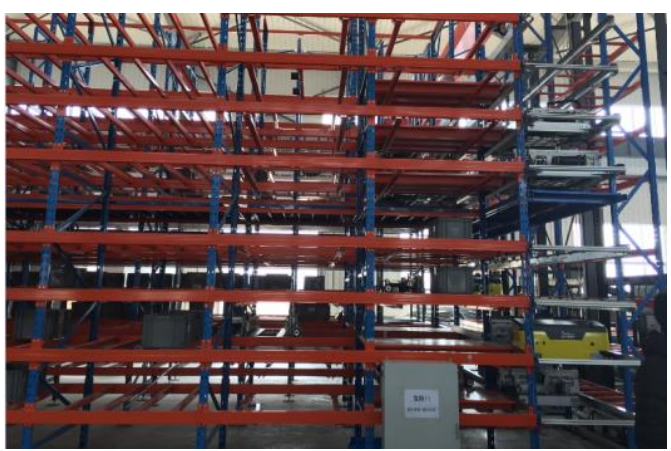

Figure 5. The Dense Channels Accessing-Sorting System

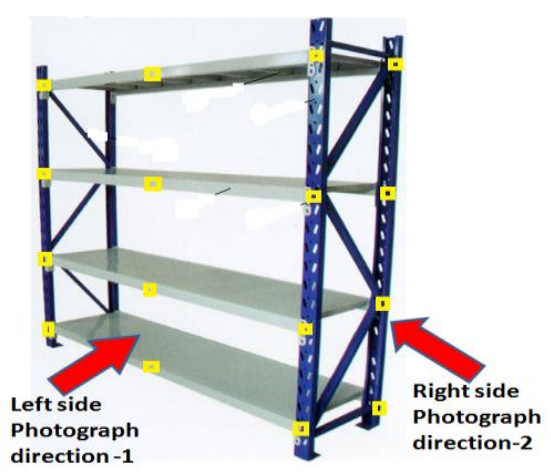

Figure 4. The Rack Model for the First Experiment

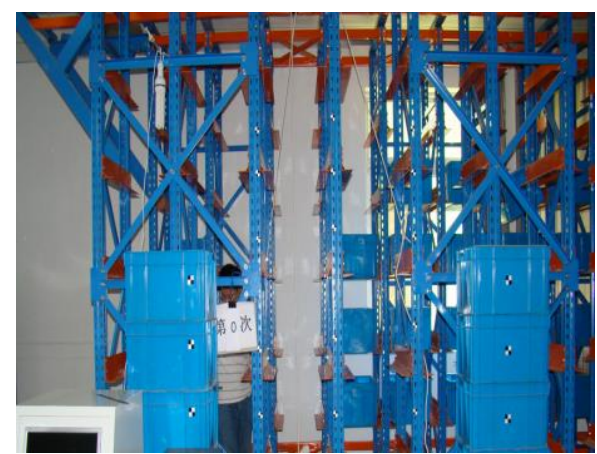

Figure 6. The Initial Image of the Second Experiment

\subsection{Dynamic Experiment}

The experiment devices are two digital cameras which have been adjusted, and two dumbbells $(20 \mathrm{~kg}, 30 \mathrm{~kg})$. Just like the first experiment, two computers installed software system which has developed by us and a printer to get the experiment results.

We take the second experiment in an automated storage\& retrieval system of one Logistic enterprise as Figure 5. Choose a corner part of the racks made up of angle steel as model, such as Figure 6. Designed pressure is $135 \mathrm{KN}$.

At first, we will let every camera take a photo before experiment as the original photos. Then we put pressure on rack slowly, use the dumbbell which impacted the rack at design angle as $20^{\circ}, 45^{\circ}, 60^{\circ}, 75^{\circ}, 90^{\circ}$, it should fall from high position to lower freely, and the mass of dumbbell is $5 \mathrm{~kg}, 10 \mathrm{~kg}, 15 \mathrm{~kg}$ step by step.

We take photos when the dynamic on goods shelf is emerged at once by the two cameras, then input the digital image into computer in time. The photo directions are set at the left side or right side of the rack (See Figure 4). 


\section{Data Analysis}

\subsection{Analysis of the First Experiment}

We compare the calculated distance by direct linear transformation formula with the high-precision measured one, and then list the data as Table 1:

Table 1. Distance of the Deformation Points

\begin{tabular}{c|c|c|c}
\hline point marks & U1- U2 $(\mathrm{mm})$ & U2-U3 $(\mathbf{m m})$ & U3- U4 $(\mathbf{m m})$ \\
\hline computed distance & 497 & 495 & 506 \\
\hline precision distance & 498 & 495 & 505 \\
\hline errors & 1 & 0 & 1 \\
\hline
\end{tabular}

From the experiment data we get the curve map of rack deformation as Figure 7 below:

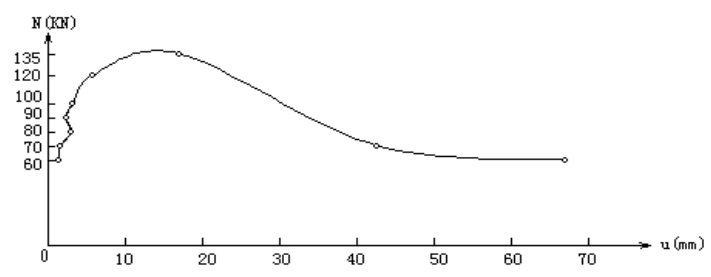

\section{Figure 7. Load-Deflection Curve Map of Deformation}

The map reflects the deformation process as the pressure is put on the rack model step by step, which is suitable for the deformation principle of the steel racks.

\subsection{Data Analysis of the Second Experiment}

With the following Table 2, we calculate the horizontal direction acceleration and vertical direction acceleration that impacted onto the rack.

Table 2. Vertical Direction Acceleration (impacted time is $\mathbf{0 . 2 s e c o n d )}$

\begin{tabular}{|c|c|c|c|c|c|}
\hline Experiment times & $\begin{array}{c}\text { Mass } \\
\text { (kg) }\end{array}$ & $\begin{array}{c}\text { Height } \\
\text { (m) }\end{array}$ & $\begin{array}{c}\text { Momentum } \\
(\mathrm{kg} \cdot \mathrm{m} / \mathrm{s})\end{array}$ & $\begin{array}{c}\text { Velocity } \\
(\mathrm{m} / \mathrm{s})\end{array}$ & $\begin{array}{l}\text { Acceleration } \\
\left(\mathbf{m} / \mathbf{s}^{2}\right)\end{array}$ \\
\hline 1 & 5 & 1.2 & 24.2487 & 4.85 & 24.25 \\
\hline 2 & 5 & 1.62 & 28.1745 & 5.63 & 28.15 \\
\hline 3 & 5 & 2.1 & 32.078 & 6.42 & 32.1 \\
\hline 4 & 10 & 1.2 & 48.4974 & 4.85 & 24.25 \\
\hline 5 & 10 & 1.62 & 56.3489 & 5.63 & 28.15 \\
\hline 6 & 10 & 2.1 & 64.1561 & 6.42 & 32.1 \\
\hline 7 & 15 & 1.2 & 72.7461 & 4.85 & 24.25 \\
\hline 8 & 15 & 1.62 & 84.5234 & 5.63 & 28.15 \\
\hline Experiment times & $\begin{array}{c}\text { Mass } \\
(\mathrm{kg})\end{array}$ & $\begin{array}{c}\text { Height } \\
\text { (m) }\end{array}$ & $\begin{array}{c}\text { Momentum } \\
(\mathrm{kg} \cdot \mathrm{m} / \mathrm{s})\end{array}$ & $\begin{array}{c}\text { Velocity } \\
(\mathrm{m} / \mathrm{s})\end{array}$ & $\begin{array}{l}\text { Acceleration } \\
\left(\mathrm{m} / \mathrm{s}^{2}\right)\end{array}$ \\
\hline 9 & 15 & 1.86 & 90.5682 & 6.04 & 30.2 \\
\hline 10 & 20 & 1.2 & 96.9948 & 4.85 & 24.25 \\
\hline 11 & 20 & 1.62 & 112.6978 & 5.63 & 28.15 \\
\hline 12 & 20 & 1.86 & 120.7576 & 6.04 & 30.2 \\
\hline
\end{tabular}


Table 3. Horizontal Direction Acceleration (impacted time is $\mathbf{0 . 2 s e c o n d )}$

\begin{tabular}{c|c|c|c|c|c}
\hline Experiment times & $\begin{array}{c}\text { Mass } \\
(\mathbf{k g})\end{array}$ & $\begin{array}{c}\text { Height } \\
(\mathbf{m})\end{array}$ & $\begin{array}{c}\text { Momentum } \\
(\mathrm{kg} \cdot \mathrm{m} / \mathbf{s})\end{array}$ & $\begin{array}{c}\text { Velocity } \\
(\mathbf{m} / \mathbf{s})\end{array}$ & $\begin{array}{c}\text { Acceleration } \\
\left(\mathbf{m} / \mathbf{s}^{2}\right)\end{array}$ \\
\hline 1 & 10 & 15 & 10.7248 & 1.07 & 5.35 \\
\hline 2 & 10 & 25 & 17.7841 & 1.78 & 8.9 \\
\hline 3 & 10 & 35 & 24.7081 & 2.47 & 12.35 \\
\hline 4 & 10 & 45 & 31.4443 & 3.14 & 15.7 \\
\hline 5 & 10 & 55 & 37.9415 & 3.79 & 18.95 \\
\hline
\end{tabular}

From Table 2 and Table 3, we can calculate the momentum and the force impacted onto the rack. Then we input the force value to computers, and the displacement of the deformation points displayed on the screen as Table 4. In the table, we give the real coordinate values of the reference points $\mathrm{C}_{0} \sim \mathrm{C}_{9}$, and deformation points $\mathrm{U}_{0} \sim \mathrm{U}_{10}$, in which the unit of the real $\mathrm{X}$ and $\mathrm{Y}$ coordinate value is $\mathrm{mm}$.

Table 4. Real Coordinate Values of the Deformation Points

\begin{tabular}{|c|c|c|c|c|c|c|c|c|c|c|c|c|c|c|c|c|}
\hline \multirow{2}{*}{$\begin{array}{l}\text { Load } \\
(\mathrm{KN})\end{array}$} & \multicolumn{2}{|c|}{17.5} & \multicolumn{2}{|c|}{30} & \multicolumn{2}{|c|}{35} & \multicolumn{2}{|c|}{40} & \multicolumn{2}{|c|}{45} & \multicolumn{2}{|c|}{50} & \multicolumn{2}{|c|}{55} & \multicolumn{2}{|c|}{60} \\
\hline & $\mathbf{X}$ & $\mathbf{Y}$ & $\mathbf{X}$ & $\mathbf{Y}$ & $\mathbf{X}$ & $\mathbf{Y}$ & $\mathbf{X}$ & $Y$ & $\mathbf{X}$ & $\mathbf{Y}$ & $\mathbf{X}$ & $\mathbf{Y}$ & $\mathbf{X}$ & $Y$ & $\mathbf{X}$ & $\mathbf{Y}$ \\
\hline $\mathrm{C}_{0}$ & 849 & 253 & 852 & 336 & 867 & 360 & 838 & 340 & 812 & 251 & 788 & 295 & 865 & 245 & 954 & 268 \\
\hline $\mathrm{C}_{1}$ & 848 & 312 & 850 & 396 & 866 & 420 & 837 & 400 & 811 & 411 & 787 & 355 & 865 & 365 & 955 & 327 \\
\hline $\mathrm{C}_{2}$ & 847 & 370 & 849 & 453 & 865 & 477 & 836 & 458 & 810 & 467 & 786 & 412 & 866 & 363 & 961 & 385 \\
\hline $\mathrm{C}_{3}$ & 843 & 434 & 844 & 516 & 860 & 541 & 831 & 522 & 805 & 532 & 781 & 477 & 859 & 428 & 949 & 446 \\
\hline $\mathrm{C}_{4}$ & 839 & 493 & 841 & 576 & 856 & 601 & 827 & 571 & 802 & 591 & 777 & 536 & 855 & 486 & 943 & 506 \\
\hline $\mathrm{C}_{5}$ & 96 & 219 & 99 & 302 & 114 & 327 & 85 & 307 & 60 & 316 & 35 & 262 & 113 & 213 & 203 & 233 \\
\hline $\mathrm{C}_{6}$ & 96 & 288 & 99 & 370 & 116 & 395 & 86 & 374 & 59 & 385 & 37 & 331 & 116 & 282 & 204 & 301 \\
\hline $\mathrm{C}_{7}$ & 96 & 363 & 100 & 445 & 114 & 470 & 85 & 450 & 60 & 461 & 37 & 405 & 116 & 356 & 205 & 376 \\
\hline $\mathrm{C}_{8}$ & 99 & 437 & 101 & 510 & 116 & 534 & 87 & 514 & 62 & 525 & 37 & 471 & 116 & 421 & 205 & 439 \\
\hline $\mathrm{C}_{9}$ & 99 & 428 & 100 & 561 & 116 & 585 & 88 & 565 & 62 & 576 & 37 & 522 & 115 & 472 & 207 & 492 \\
\hline $\mathbf{U}_{0}$ & 583 & 157 & 585 & 239 & 600 & 264 & 571 & 244 & 546 & 255 & 522 & 198 & 599 & 149 & 689 & 169 \\
\hline $\mathbf{U}_{1}$ & 582 & 227 & $\mathbf{5 8 5}$ & 311 & 599 & 335 & 570 & 314 & 545 & 325 & 522 & 269 & 599 & 220 & 687 & 238 \\
\hline $\mathbf{U}_{2}$ & 580 & 320 & 582 & 402 & 597 & 407 & 568 & 407 & 543 & 418 & 516 & 362 & $\mathbf{5 9 8}$ & 312 & 687 & 331 \\
\hline $\mathbf{U}_{3}$ & 577 & 411 & 579 & 494 & 594 & 518 & 565 & 499 & 540 & 519 & 516 & 452 & 595 & 403 & 685 & 442 \\
\hline $\mathbf{U}_{4}$ & 576 & 496 & 578 & 578 & 595 & 603 & 565 & $\mathbf{5 8 5}$ & 539 & 593 & 515 & 536 & 595 & 487 & 685 & $\mathbf{5 0 8}$ \\
\hline $\mathbf{U}_{5}$ & 554 & 501 & 556 & 580 & 571 & 607 & 542 & 586 & 517 & 598 & 495 & 542 & 573 & 491 & 660 & 511 \\
\hline $\mathbf{U}_{6}$ & 480 & 494 & 482 & 578 & 498 & 602 & 469 & 581 & 443 & 593 & 418 & $\mathbf{5 3 7}$ & 498 & 488 & 586 & $\mathbf{5 0 7}$ \\
\hline $\mathbf{U}_{7}$ & 452 & 486 & 454 & 569 & 470 & 594 & 441 & 574 & 415 & 584 & 392 & 529 & 471 & 479 & 559 & 500 \\
\hline $\mathbf{U}_{8}$ & 454 & 403 & 456 & 487 & 472 & 511 & 442 & 492 & 416 & 502 & 393 & 447 & 471 & 398 & 560 & 416 \\
\hline $\mathbf{U}_{9}$ & 555 & 320 & 556 & 403 & 572 & 427 & 544 & 407 & 417 & 419 & 494 & 363 & 473 & 314 & 658 & $\mathbf{3 3 3}$ \\
\hline $\mathbf{U}_{10}$ & 483 & 318 & 485 & 402 & 501 & 425 & 472 & 306 & 446 & 415 & 422 & 359 & 499 & 310 & $\mathbf{5 8 8}$ & 329 \\
\hline
\end{tabular}

There are so much data collected from the second experiment, so we can't display all of them, we only cite some points which distort more obvious relatively as displayed in Figure 8 . 


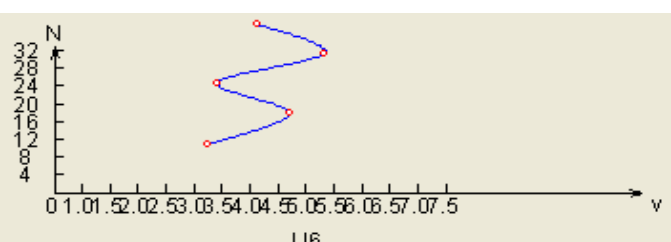

U6

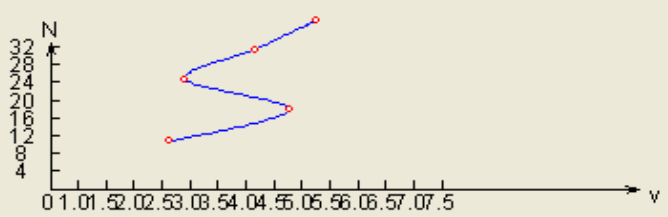

U7

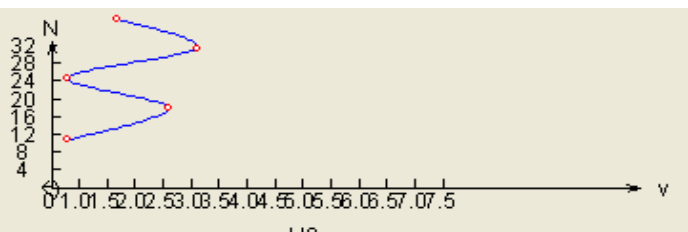

บ9

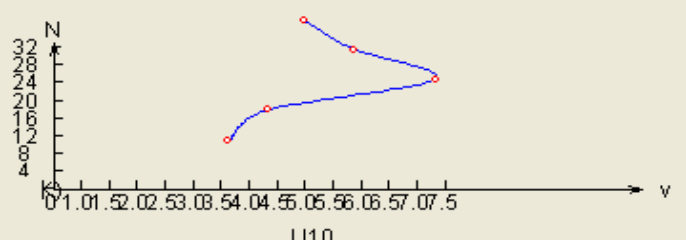

U10

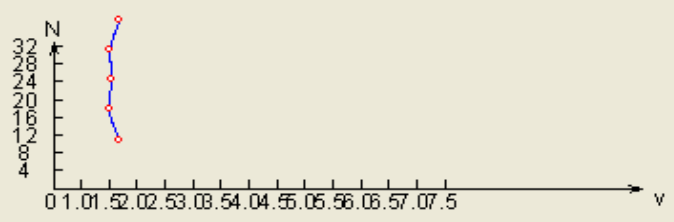

บ8

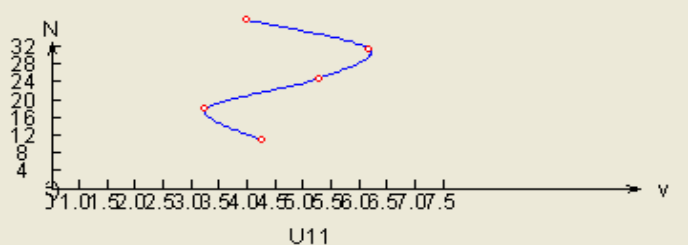

U11

Figure 8. The Deformation Map of some Points

\section{Main Conclusions}

As the very import part in the DCASS, the racks are almost made of steel structure. For different kind of steel structure, the force of the steel and the performance requirements are also different. It is often accompanied by large deformation of plastic failure when the steel is damaged in force. Some cases also may be happened suddenly without any sign and obvious deformation of the brittle failure.

From the results and the deformation maps of some points in the vertical direction, we can find that steel racks' deformation vary within the elastic limit, the deformation of each point is always swinging around the center. So in the vertical direction of the experiment, the racks have deformation in the whole experiment process, but eventually returned to the original position. The racks can bear the brunt of the design standards.

For the various points in the direction of horizontal, the characteristics appear the same as the points in the vertical direction around the center, shows that the racks are stable.

There is no damage occur overall the impact of racks in the experiment. This proves the racks are stable and qualifies the rightness of the racks' displacement analysis and stability analysis. The racks can give the security and stability of items in the big earthquake motions.

The experiments show that we can obtain high precision deformation results up to $2.3 \%$ by using DCRP method. The precision result can satisfy the requirements for monitoring the deformation of racks in AS/RS systems [12-13]. The monitoring method will benefit the Logistic Warehouses such as the DCASS and others, and also bring the safety to our world when they are put in practice.

Though restricted by the properties of digital camera, and also there are some waves in the result data, we will get higher precision result in the future study when using higher resolution, larger scope optical zoom, and better optical lens digital cameras.

The DCRP method also can be applied to monitor the deformation on the large structure such as a bridge, a steel tower, and tall buildings [14]. The method can give us a convenient, cheap, and precise way to monitor the structures instantaneously, dynamically and real time.

It is difficult to reach or realize real-time monitoring during the construction process of the machinery and buildings. By using the DCRP method for the deformation monitoring, we can realize the process: data acquisition, processing and graphical display function. 
Except above, we also can make the operation more convenient, efficient, and the digital cameras can be flexible placed with a certain angle, and the requirements is more looser for actual monitoring site, and make it easy to implement a remote monitoring work. By using pixels of more than 4 million digital cameras and data processing system, we can do measurement work in 60 meters.

\section{Acknowledgment}

Project supported by the National Nature Science Foundation of China (No. 61304008), Shandong Province Major Scientific Research Projects (2010GX20125), and Doctor's Foundation of Shandong Jianzhu University (XNBS:1338).

Thanks very much to my doctoral supervisor-Professor Yaohua Wu, and also thanks very much to my doctoral supervisor off-campus_-Professor Chengxin Yu.

\section{References}

[1] R. Hamberg and J. Verriet, "Automation in Warehouse Development", Springer-Verlag London Limited (2012). pp. 3-79.

[2] W. Zhang and Y. Pu, "Logistics system Management on Self Organization", Journal of Transportation Engineering and Information, vo1. 2, no. 1, (2004) March, pp. 11-87.

[3] J. Gu, M. Goetschalckx and L. F. McGinnis, "Research on warehouse design and performance evealuation: A comprehensive review", Europe Journal of Operation Research, vol. 203, (2010), pp. 539-549.

[4] E. Frazelle, "World-class warehousing and material handling", McGraw-Hill, Columbus, (2002).

[5] E. Hassini, "One-dimensional carousel storage problems: applications, review and generalizations", INFOR, vol. 47, no. 2, (2009), pp. 81-92.

[6] M. Vlasiou, I. J.-B. F. Adan and J. Wessels, “A Lindley-type equation arising from a carousel problem”, Journal of Applied Probability, vol. 41, (2004), pp. 1171-1181.

[7] E. Hassini and R. G. Vickson, "A two-carousel storage location problem", Computers and Operations Research, vol. 30, (2003), pp. 527-539.

[8] F. Wenhao, "Close-Range Photogrammetry", Wuhan Technical University of Surveying and Mapping", (2000).

[9] Q. Luqian and L. Shaopu, "Steel Structure", Earthquake Publishing House, (1991).

[10] J. Zhengxian and C. Xijing, "Strength Calculation and Design of AS\&RS goods shelf", Logistics Technology, vol. 3, (2003), pp. 72-75.

[11] J. Stable and R. Mingmin, "Fluid-structure-interaction for the analysis of the dynamics of fuel storage racks in the case of seismic loads", Nuclear Engineering and Design, (2001), pp. 167-176.

[12] M. Chen, C. Yu, N. Xu and Y. Zhao, "Application Study of Digital Analytical Method on Deformation Monitor of high-rise Goods Shelf", Proceedings of the IEEE International Conference on Automation and Logistics Qingdao, China, (2008) September, pp. 2084-2088.

[13] C. AguirTe, "Seismic behavior of rack structures", Journal of Constructional Steel Research, (2005), pp. 607-624.

[14] T. Hara and M. Nakabo, "Dynamic Deformation Measurement of Real Bridge Using Sampling Moiré Camera", Proceedings of the IEEE International Conference on Intelligent Computing and Integrated Systems, (2011).

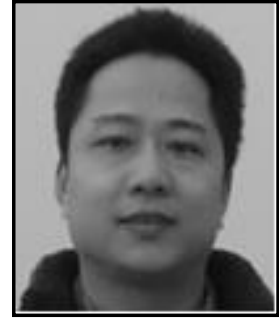

Yongqian Zhao, (1972.07) - $\mathrm{He}$ is a $\mathrm{PhD}$ candidate student in School of Control Science and Engineering, Shandong University, Jinan, China. And also an associate professor in the School of Natural Science, Shandong Jianzhu University. His research interests include Systematical Engineering and Logistic Optimization. Email address:13455111419@163.com ; Tel.:+86 13455111419. 


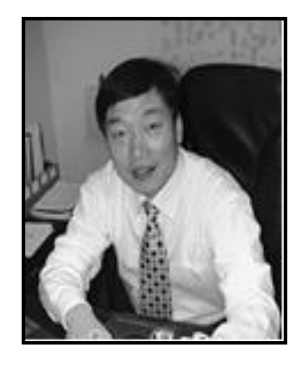

Yaohua Wu, (1963.09) - He is currently a professor and doctoral tutor at School of Control Science and Engineering, Shandong University, China. He received his $\mathrm{PhD}$ degree from Tsinghua University, China. He was a vice president in China Society of Logistics (CSL), a director in Chinese Mechanical Engineering Society, and a senior member in America Society of Manufacturing Engineers (ASME). His research interests include the Designing and Simulation of Integration Logistics System, The Automated Sorting System and Logistics Equipment Technology and Theory, etc..Email: yaohua.wu@sdu.edu.cn; Tel: +86-531-88392269.)

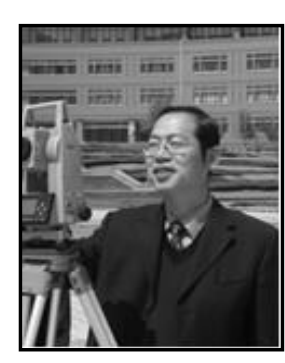

Chengxin Yu, (1961.11) - He is the second-class professor of Shandong Jianzhu University, doctoral tutor, graduated from Wuhan University. And he was prized special government allowances by the State Council. He is Shandong province outstanding contribution young and middle-aged experts. His research interests include Systematical Engineering and Logistic optimization, Digital CloseRange Photogrammetric Technology, etc..Email address: ycx1108@126.com; Tel.:+86 13506412501. 